\title{
Constitutive redox and phosphoproteome changes in multiple herbicide resistant Avena fatua $L$. are similar to those of systemic acquired resistance and systemic acquired acclimation
}

\section{Authors: Erin E. Burns, Barbara K. Keith, Refai Y. Mohammed, Brian Bothner, and William E. Dyer}

NOTICE: this is the author's version of a work that was accepted for publication in Journal of Plant Physiology. Changes resulting from the publishing process, such as peer review, editing, corrections, structural formatting, and other quality control mechanisms may not be reflected in this document. Changes may have been made to this work since it was submitted for publication. A definitive version was subsequently published in Journal of Plant Physiology, v. 220, January 2018, DOI\# 10.1016/i.jplph.2017.11.004

Burns, Erin E. , Barbara K. Keith, Refai Y. Mohammed, Brian Bothner, and William E. Dyer. "Constitutive redox and phosphoproteome changes in multiple herbicide resistant Avena fatua $\mathrm{L}$. are similar to those of systemic acquired resistance and systemic acquired acclimation." Journal of Plant Physiology 220 (November 2017): 105-114. DOI: 10.1016/j.jplph.2017.11.004.

Made available through Montana State University's $\underline{\text { ScholarWorks }}$ 


\title{
Constitutive redox and phosphoproteome changes in multiple herbicide resistant Avena fatua L. are similar to those of systemic acquired resistance and systemic acquired acclimation
}

\author{
Erin E. Burns ${ }^{\mathrm{a}}$, Barbara K. Keith ${ }^{\mathrm{a}}$, Mohammed Y. Refai ${ }^{\mathrm{b}}$, Brian Bothner ${ }^{\mathrm{b}}$, William E. Dyer ${ }^{\mathrm{a}, *}$ \\ a Department of Plant Sciences \& Plant Pathology, PO Box 173150, Montana State University, Bozeman, MT 59717, United States \\ ${ }^{b}$ Department of Chemistry \& Biochemistry Research, PO Box 173400, Montana State University, Bozeman, MT 59717, United States
}

\begin{abstract}
A B S T R A C T
Plants are routinely confronted with numerous biotic and abiotic stressors, and in response have evolved highly effective strategies of systemic acquired resistance (SAR) and systemic acquired acclimation (SAA), respectively. A much more evolutionarily recent abiotic stress is the application of herbicides to control weedy plants, and their intensive use has selected for resistant weed populations that cause substantial crop yield losses and increase production costs. Non-target site resistance (NTSR) to herbicides is rapidly increasing worldwide and is associated with alterations in generalized stress defense networks. This work investigated protein post-translational modifications associated with NTSR in multiple herbicide resistant (MHR) Avena fatua, and their commonalities with those of SAR and SAA. We used proteomic, biochemical, and immunological approaches to compare constitutive protein profiles in MHR and herbicide susceptible (HS) A. fatua populations. Phosphoproteome and redox proteome surveys showed that post-translational modifications of proteins with functions in core cellular processes were reduced in MHR plants, while those involved in xenobiotic and stress response, reactive oxygen species detoxification and redox maintenance, heat shock response, and intracellular signaling were elevated in MHR as compared to HS plants. More specifically, MHR plants contained constitutively elevated levels of three protein kinases including the lectin S-receptor-like serine/threonine-protein kinase LecRK2, a well-characterized component of SAR. Analyses of superoxide dismutase enzyme activity and protein levels did not reveal constitutive differences between MHR and HS plants. The overall results support the idea that herbicide stress is perceived similarly to other abiotic stresses, and that $A$. fatua NTSR shares analogous features with SAR and SAA. We speculate that MHR A. fatua's previous exposure to sublethal herbicide doses, as well as earlier evolution under a diversity of abiotic and biotic stressors, has led to a heightened state of stress preparedness that includes NTSR to a number of unrelated herbicides.
\end{abstract}

\section{Introduction}

Plants are regularly confronted with numerous external stresses from biotic and abiotic sources during their evolution. To combat biotic invasions by pathogenic bacteria and fungi, they have evolved highly effective innate and induced immune responses (Dodds and Rathjen, 2010). The components and pathways of pattern-triggered immunity (PTI) (Boller and Felix, 2009) and systemic acquired resistance (SAR) (Fu and Dong, 2013) are well characterized, involving recognition, signal transduction, and transcriptional reprogramming in response to biotic challenge.

Similarly, plants have evolved related strategies to acclimate to abiotic stresses such as heat, cold, and salt. Systemic acquired acclimation (SAA) describes an accumulation of transcripts and proteins that confer enhanced resistance to subsequent abiotic stresses (Suzuki et al., 2013). Although less detail is known about SAA than SAR, there are a number of commonalities between the two (Atkinson and Urwin, 2012; Fujita et al., 2006). For example, both systems include stress recognition proteins such as receptor-like kinases (RLKs) and lectin receptor kinases (LRKs), and include key roles for reactive oxygen species (ROS), overall cellular redox state, and mitogen-activated protein (MAP) kinase-mediated signal transduction cascades (Mittler and Blumwald, 2015).

A much more evolutionarily recent abiotic stress imposed on plants is the application of herbicides. Herbicides are obviously designed to control plants, but they can also merely cause injury, due to sublethal 
dosages (improper applications or equipment problems), adverse environmental conditions that reduce efficacy, or delayed applications on older, more tolerant plants (Caseley and Walker, 1990). Under these conditions, susceptible plants respond much the same as they do to other abiotic stresses (reviewed in (Alberto et al., 2016)). Both abiotic (Dietz et al., 2016) and herbicide stresses (Dayan and Watson, 2011; Sewelam et al., 2016) induce rapid ROS generation, and even though herbicides are designed to inhibit a specific biochemical target, both impact multiple pathways and cellular components (Délye, 2013; Suzuki et al., 2014). Plant transcriptome changes caused by herbicide stress are quite similar to those resulting from abiotic and biotic stresses (Das et al., 2010; Unver et al., 2010).

Worldwide intensive herbicide use has led to the evolution of resistant weed populations that cause substantial crop yield losses and increase production costs (Heap, 2014). Resistance can be conferred by target site overexpression or mutations that alter herbicide binding, or non-target site resistance (NTSR) mechanisms like enhanced rates of herbicide metabolism, reduced absorption and/or translocation, sequestration, or more generalized stress defense networks (Délye, 2013). Selection of herbicide resistant (HR) plants is thought to operate on standing genetic variation as affected by biological (species characteristics) and operational (herbicide dose and use patterns) factors (Georghiou and Taylor, 1986; Jasieniuk et al., 1996). NTSR evolves gradually, appears to be controlled by multiple genes, and is conferred by one or more constitutive and/or induced physiological mechanisms (reviewed in (Délye, 2013)). Recent transcriptome analyses of constitutive changes in HR or multiple herbicide resistant (MHR) populations show that transcripts representing functions in xenobiotic metabolism and stress response are more abundant prior to herbicide treatment (Gaines et al., 2014; Hofer et al., 2014; Peng et al., 2010).

We recently described populations of MHR Avena fatua L. that are resistant to members of all selective herbicide families available in the U.S. for A. fatua control in small grain crops (Keith et al., 2015; Lehnhoff et al, 2013). MHR3 and MHR4 plants do not contain known target site mutations for acetolactate synthase (ALS) or acetyl-CoA carboxylase (ACCase) inhibitors, and the cytochrome P450 monooxygenase (P450) inhibitor malathion partially reversed the resistance phenotype for several herbicides (Keith et al., 2015), indicating that NTSR mechanisms are involved. Transcriptome and proteome analyses show that MHR A. fatua plants have constitutively altered levels of stress-related differentially expressed genes (DEGs) and proteins (Keith et al., 2017). Specifically, DEGs and proteins with functions in xenobiotic catabolism, stress response, redox maintenance, and transcriptional regulation are constitutively elevated in untreated MHR plants. We now extend these transcriptional and translational investigations to include surveys of post-translational modifications (PTMs) including protein phosphorylation and redox state of individual proteins.

\section{Materials and methods}

\subsection{Plant material}

The MHR3 and MHR4 populations were derived from seeds collected in 2006 from two $A$. fatua populations not controlled by $60 \mathrm{~g} \mathrm{a} \mathrm{i} \mathrm{ha}^{-1}$ pinoxaden (Axial, Syngenta Crop Protection; ACCase inhibitor) in two production fields separated by approximately $8 \mathrm{~km}$ in Teton County, Montana, USA. Field-collected seeds (about $90 \%$ of which were resistant to $60 \mathrm{~g}$ a i ha ${ }^{-1}$ pinoxaden, data not shown) were subjected to two generations of recurrent group selection (50 plants each generation) by spraying with the same dose of pinoxaden (for MHR3 and MHR4) or surfactant only (susceptible populations), followed by five additional generations with no herbicide selection in the greenhouse. From each generation of 50 plants, all seeds were harvested and a random selection of 50 seeds was used to initiate the next generation. The herbicide susceptible population HS1 was derived from seeds collected from untreated border plants in an adjacent field, and was subsequently confirmed to be $100 \%$ susceptible to the herbicides used in these studies (Keith et al., 2015; Lehnhoff et al., 2013). A second susceptible population, HS2, is the inbred nondormant SH430 line used in seed dormancy research (Johnson et al., 1995; Naylor and Jana, 1976). Plants were grown under a 16-h photoperiod of natural sunlight supplemented with mercury vapor lamps $\left(165 \mu \mathrm{mol} \mathrm{m}^{-2} \mathrm{sec}^{-1}\right)$ at $25 \pm 4 \mathrm{C}$ in standard greenhouse soil mix [1:1:1 (by vol) Bozeman silt loam:Sunshine mix \#12 (Sun Gro Horticulture, Inc., Bellvue, WA):perlite] and fertilized weekly with Jack's water soluble $20 \mathrm{~N}-20 \mathrm{P}-20 \mathrm{~K}$ fertilizer (JR Peters Inc., Allentown, PA). Plants for each experiment were grown on the same greenhouse bench and were harvested in midmorning to minimize potential environmental- and circadian-related protein changes.

\subsection{Protein extraction}

For phosphoproteome analysis, shoot tissue from three replicate three-leaf stage HS1 and MHR4 plants was harvested, ground under liquid nitrogen, and $200 \mathrm{mg}$ of tissue from each plant was suspended in ice cold extraction buffer containing $0.1 \mathrm{M}$ Tris $\mathrm{HCl}(\mathrm{pH} 7.5), 2 \mathrm{mM}$ EDTA, $1 \mathrm{mM}$ DTT, $1 \mathrm{mM}$ PMSF, and 5\% (w/v) PVPP. The slurries were filtered through Miracloth (EMD Millipore, Merck KGaA, Darmstadt, Germany) and filtrates were centrifuged at $21,380 \mathrm{xg}$ for $10 \mathrm{~min}$ at $4 \mathrm{C}$. Proteins were concentrated by precipitation with four volumes of icecold acetone containing $10 \%$ TCA at $-80 \mathrm{C}$ overnight. Two additional $100 \%$ acetone precipitations were performed for $3 \mathrm{~h}$ each at $-80 \mathrm{C}$, and proteins were resuspended in two-dimensional polyacrylamide gel electrophoresis (2D-E) buffer ( $30 \mathrm{mM}$ Tris $\mathrm{pH} 8.5,7 \mathrm{M}$ urea, $2 \mathrm{M}$ thiourea, 4\% CHAPS, $1 \mathrm{x}$ protease inhibitor/nuclease mix [GE Healthcare Life Sciences, Pittsburgh, PA], and $0.1 \%$ [w/v] bromophenol blue) to a final concentration of $2 \mathrm{mg} \mathrm{mL}^{-1}$. Protein concentrations were determined (Bradford, 1976) using bovine serum albumin fraction $\mathrm{V}$ as standard.

For redox proteome analysis, shoot tissue from three replicate threeleaf stage HS1 and MHR4 plants was extracted as described above and subjected to the blocking 5-(iodoacetamido)fluorescein (IAF) labeling method described in Wang et al. (2011). Thiol groups of oxidized proteins were labeled with Rhodamine $\operatorname{Red}^{\circ} \mathrm{C}_{2}$ maleimide (Thermo Fisher Scientific, Waltham MA) in a final concentration of $40 \mu \mathrm{M}$ during a 30-min dark incubation at room temperature, and then resuspended in 2D-E buffer to a final concentration of $2 \mathrm{mg} \mathrm{mL}^{-1}$ (Waszczak et al., 2015). Protein concentrations were determined as above.

\subsection{D electrophoresis}

Extracts containing 150 or $50 \mu \mathrm{g}$ of protein for phosphoproteome or redox proteome analysis, respectively, were diluted to a final volume of $450 \mu \mathrm{L}$ with isoelectric focusing (IEF) buffer (2D-E buffer containing $50 \mathrm{mM}$ DTT and 0.5\% (v/v) IPG buffer 3-11 NL [GE Healthcare]), and incubated for $1 \mathrm{~h}$ at room temperature. Three replicate extracts each from MHR4 or HS1 shoots were separately loaded on IPG strips (pH 3$11 \mathrm{NL}$, non-linear, $24 \mathrm{~cm}$ length [GE Healthcare]), and IEF and SDSPAGE were carried out as described by Maaty et al. (2012).

\subsubsection{Image acquisition and analysis}

After electrophoresis, phosphoproteome gels were stained with the fluorescent phosphoprotein specific stain Pro-Q Diamond (Invitrogen Corp. Carlsbad, CA), which detects phosphate groups attached to tyrosine, serine, or threonine residues, following the methods of Agrawal and Thelen (2005). Gels were scanned with a Typhoon Trio Imager following the Invitrogen protocol. Redox proteome gels were scanned with the same imager using the Thermo Fisher Scientific protocol. All gels were scanned at $100 \mu \mathrm{m}$ resolution and $640 \mathrm{~V}$ for PMT. Images were subjected to an automated in-gel analysis using Progenesis SameSpots v 3.0.2 software (Nonlinear Dynamics Ltd. Newcastle, UK), which aligns multiple gel images while adjusting for positional 


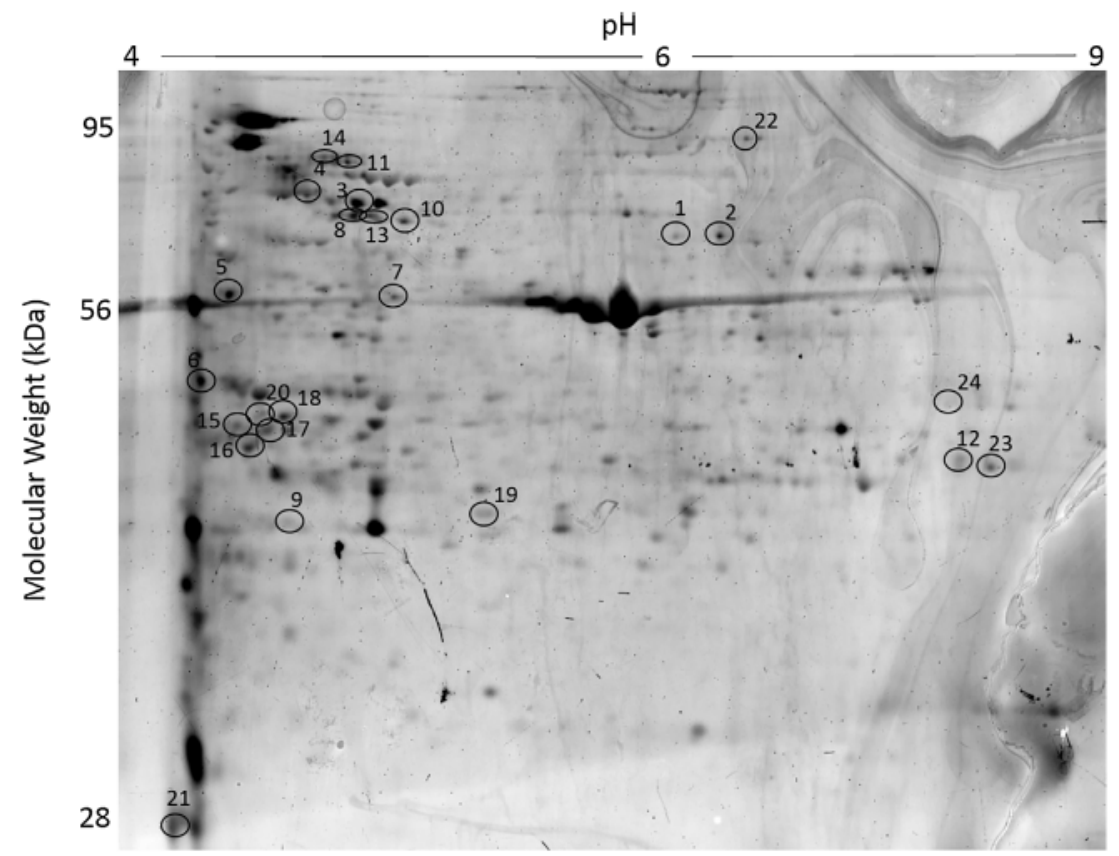

Fig. 1. Representative two dimensional electrophoresis gel of the multiple herbicide resistant (MHR4) Avena fatua phosphoproteome. Significantly differential (between MHR and herbicide susceptible (HS1) plants) protein spots were numbered, excised from gels, and identified by LC MS/MS. Spot numbers are the same as in Table 1.

variations so that individual protein spots can be compared among samples. Individual spot volumes were calculated for multivariate statistical analysis. Gels used for protein identification each contained $400 \mu \mathrm{g}$ of protein and were stained with colloidal coomassie stain (Dyballa and Metzger, 2009), destained in 10\% (v/v) acetic acid, and stored at $4 \mathrm{C}$ in $1 \%(\mathrm{v} / \mathrm{v})$ acetic acid until spot excision.

\subsubsection{Protein mass determination and analysis}

After electrophoresis, all significantly differential (p-value $<0.1$ identified by Progenesis SameSpots) protein spots were excised, digested with porcine trypsin (Promega Corp. Madison, WI), and eluted as described in Shevchenko et al. (2006). The resulting peptides were subjected to mass analysis performed on Bruker maXis Impact with Dionex 3000 nano-uHPLC controlled with Chromeleon Xpress (2.13 for Hystar) following the methods of Maaty et al. (2012).

Protein identification followed the methods of Mason et al. (2016), initiated by generating a custom protein sequence file (.FASTA) from the Oryza sativa subsp. japonica (retrieved 2016; http://www.UniProt. org; containing 121,989 entries) and Brachypodium distachyon (retrieved 2016; http://www.UniProt.org; containing 50,507 entries) databases. Sequences for the potential contaminants human keratin (retrieved 2016; http://www.UniProt.org; containing 49 entries) and porcine trypsin (retrieved 2016; http://www.UniProt.org; containing 1 entry) were added to the sequence file to create a final target-decoy library. The library was queried against data files using the following search parameters: up to two missed cleavages allowed; precursor charges $+2,+3,+4$; precursor ion mass tolerance $30 \mathrm{ppm}$; and fragmentation mass tolerance of $0.5 \mathrm{Da}$. Post translational modifications were defined as oxidation of $\mathrm{M}$, acetylation of $\mathrm{N}$-terminus, and carboxylation of C-terminus (defined in SearchGUI software; Vaudel et al. (2011)). Two or more significant peptides with an FDR $\leq 1.0 \%$ (PeptideShaker software (Vaudel et al., 2015)) were required for annotation of each protein from the PaxDb database (Wang et al., 2012) as accessed through UniProtKB and the Rice Genome Annotation Project (Kawahara et al., 2013).

\section{4. $1 D$ page}

Protein extracts ( $20 \mu \mathrm{g}$ per lane) from HS1, HS2, MHR3, and MHR4 plants obtained as described above were heated at $95 \mathrm{C}$ for $5 \mathrm{~min}$ in $1 \mathrm{x}$ Laemmli SDS sample buffer (Cleveland et al., 1977) before being subjected to SDS-PAGE in $12.5 \%$ gels at $2.5 \mathrm{~mA} \mathrm{~cm}^{-1}$ for $3 \mathrm{~h}$ with water cooling.

\subsection{Immunoblots}

Proteins were electroblotted from SDS gels onto PVDF membrane (Pall Corporation, Radnor, PA) and probed with rabbit polyclonal antisera against Spinacia oleracea cytosolic or chloroplastic CuZn SODs (Kanematsu and Asada, 1990) diluted 1:5000 (v/v) in blotto (10\% [w/ v] nonfat dry powdered milk/phosphate-buffered saline $/ 0.001 \%[\mathrm{w} / \mathrm{v}]$ sodium azide) followed by detection with goat anti-rabbit IgG/alkaline phosphatase (Sigma-Aldrich AP132A) diluted 1:10,000 (v/v) in blotto. Densitometric analyses of immunoblot signals were performed using ImageJ (Schneider et al., 2012).

\subsection{Superoxide dismutase activity gels}

Proteins extracts ( $20 \mu \mathrm{g}$ per lane) from HS1, HS2, MHR3, and MHR4 plants obtained as described above were subjected to native electrophoresis in $12.5 \%$ polyacrylamide gels at $2.5 \mathrm{~mA} \mathrm{~cm}^{-1}$ with water cooling. Superoxide dismutase (SOD) activity was detected photochemically as described by Chen and Pan (1996) in gels after illumination with $30 \mu \mathrm{mol} \mathrm{m}^{-2} \mathrm{~s}^{-1}$ fluorescent light for $5 \mathrm{~min}$. Densitometric quantification of SOD activity bands were performed using ImageJ (Schneider et al,, 2012).

\section{Results and discussion}

2D electrophoresis was used to compare constitutive profiles of soluble phosphorylated (numbered 1-24 in Fig. 1 and Table 1) and redox-sensitive (numbered 25-47 in Fig. 2 and Table 2) proteins from untreated MHR4 and HS1 plants. Statistical analysis of phosphoproteome gels identified 24 differential protein spots, of which 14 proteins were more abundant in MHR4 than HS1 plants. Analysis of redox proteome gels identified 23 differential spots, with 15 proteins at higher levels in MHR4 plants. For both phosphoproteome and redox proteome gels, differences in fluorescent signals between MHR4 and HS1 samples were due to either altered protein amounts or changes in the number of labeled residues on individual proteins.

Differential signals from phosphorylated and redox-sensitive proteins are discussed below in five functional categories: core cellular 
Table 1

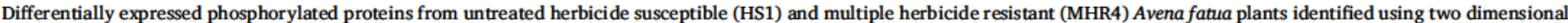
polyacrylamide gel electrophoresis.

\begin{tabular}{|c|c|c|c|c|c|c|c|c|}
\hline Function & Spot no. & Uniprot no. & Oryza sativa no. & Annotation & $\mathrm{FC}^{\mathrm{a}}$ & P-value & Peptides $^{\mathrm{b}}$ & Peptide sequences \\
\hline \multirow{27}{*}{$\begin{array}{l}\text { Core cellular } \\
\text { processes }\end{array}$} & 6 & P14655 & Os04g0659100 & Glutamine synthetase & 7.4 & 0.04 & 3 & TISKPVEDPSELPK \\
\hline & & & & & & & & WNYDGSSTGQAPGEDSEVILYPQAIFK \\
\hline & & & & & & & & WNYDGSSTGQAPGEDSEVIL YPQAIFKDPFR \\
\hline & 7 & I1 HI10 & Os10g0355800 & ATP synthase CF1 beta subunit & 2.1 & 0.06 & 4 & FVQAGSEVSALLGR \\
\hline & & & & & & & & IFNVLGEPVDNLGPVDSSATFPIHR \\
\hline & & & & & & & & TVLIMELINNIAK \\
\hline & & & & & & & & YKELQDIIAILGLDELSEEDRLTVAR \\
\hline & 14 & Q5VNW1 & Os06g0133800 & Transketolase & 3.0 & 0.05 & 2 & IKVTTTIGFGSPNKANSYSVH \\
\hline & & & & & & & & QKDTPEERNVRFGVREHGMGAICNGIALHSPGL \\
\hline & 15 & Q6ATP4 & Os11g0656500 & Putative polyprotein & -5.2 & 0.02 & 2 & DVAPSEEDRPHRKVQRPVYFVSEALWDAK \\
\hline & & & & & & & & EWTPAPEPVSVPEASSGPSQLPHTAHWVMQFD \\
\hline & 16 & Q8LH82 & Os07g0446800 & Hexokinase & -2.4 & 0.07 & 2 & EGCACAAPPAAAAPPMPK \\
\hline & & & & & & & & RMVVEVCDIVATRAARLAAAGIV \\
\hline & 17 & Q40677 & Os11g0171300 & $\begin{array}{l}\text { Fructose-bisphosphate aldolase, } \\
\text { chloroplastic }\end{array}$ & -2.5 & 0.05 & 2 & LASIGLENTEANR \\
\hline & & & & & & & & TVVSIPNGPSELAVK \\
\hline & 18 & B9F813 & Os04g0234600 & Sedoheptulose-1,7-bisphosphatase & -5.9 & 0.08 & 2 & LLFEVAPLGFLIEK \\
\hline & & & & & & & & TNFTVGTIFGVWPGDKLTGVTGGDQ \\
\hline & 19 & B8XEK6 & Os06g0133000 & Granule-bound starch synthase I & -7.3 & 0.00 & 2 & RFPSVVVYATGAGMNVVFVGAEMAPWSK \\
\hline & & & & & & & & APRINLNNNPYFKGTSGEDVVFVCNDWH \\
\hline & 21 & Q0IPE5 & Os12g0210800 & $\begin{array}{l}\text { 2-dehydro 3- } \\
\text { deoxyphosphooctonate aldolase }\end{array}$ & -2.4 & 0.06 & 3 & LYGQLKAAQPFFLLAGPN \\
\hline & & & & & & & & PVVTDVHESHQCEAVGRV \\
\hline & & & & & & & & EWLREANCPVVADVTHALQQPAGK \\
\hline & 22 & I1 J387 & Os04g0672200 & Poly(ADP-ribose) polymerase & -2.5 & 0.02 & 2 & $\begin{array}{l}\text { SKDFLFVRDLFLSGMGSFATENSIL } \\
\text { LLETLKKLHYCPSLWNKSSIEVMSS }\end{array}$ \\
\hline & 23 & Q2QSR7 & Os12g0420200 & $\begin{array}{l}\text { NAD(P)-binding domain } \\
\text { containing protein }\end{array}$ & -2.6 & 0.05 & 2 & APITQQLPGESDAEYAEFSSK \\
\hline & & & & & & & & VKDLATAFVLALGNPKASKQVFNIS \\
\hline & 24 & Q7XPR2 & Os04g0623800 & Aminomethyltransferase & -7.8 & 0.01 & 2 & LAGAAEAAEAELKKTALYDFHVAHG \\
\hline & & & & & & & & LEKSEGKVRLTGLGARDSLRLEAG \\
\hline \multirow{4}{*}{$\begin{array}{l}\text { ROS and redox } \\
\text { maintenance }\end{array}$} & 2 & P0C5D1 & Os07g0638400 & 1-Cys peroxiredoxin & 2.4 & 0.04 & 2 & GLTLGDVVPDLELDTTHGK \\
\hline & & & & & & & & ALHIVGPDKKVKLSFLFPACTGRNMAEVL \\
\hline & 11 & B7ERQ1 & Os07g0638300 & Peroxiredoxin & 3.6 & 0.06 & 2 & STHGKIRIHDFVGDTY \\
\hline & & & & & & & & VRAVDALQTAAKHAVATPVNW \\
\hline \multirow{25}{*}{$\begin{array}{l}\text { Chaperones and } \\
\text { heat shock }\end{array}$} & 3 & Q10NA9 & Os03g0276500 & $70 \mathrm{kDa}$ heat shock protein & 3.2 & 0.04 & 7 & ATAGDTHLGGEDFDNR \\
\hline & & & & & & & & DAGVISGLNVMR \\
\hline & & & & & & & & NQVAMNPINTVFDAK \\
\hline & & & & & & & & STVHDVVLVGGSTR \\
\hline & & & & & & & & TTPSYVAFTDSER \\
\hline & & & & & & & & VQDLLLLDVTPLSQGLETAGGVMTVLIPR \\
\hline & & & & & & & & VQQLLQDFFNGK \\
\hline & 5 & Q2QU06 & Os12g0277500 & $60 \mathrm{kDa}$ chaperonin alpha subunit & 6.6 & 0.03 & 9 & AVLQDIAIVTGAEFLAK \\
\hline & & & & & & & & GILNVAAIKAPSFGER \\
\hline & & & & & & & & GYISPQFVTNLEK \\
\hline & & & & & & & & LANAVGVTLGPR \\
\hline & & & & & & & & NVVLDEYGSPK \\
\hline & & & & & & & & NVVLDEYGSPKVVNDGVTIAR \\
\hline & & & & & & & & VGAATETELEDR \\
\hline & & & & & & & & VTIHQTTTTLIADAASKDEIQAR \\
\hline & & & & & & & & VVNDGVTIAR \\
\hline & 8 & Q7G2N7 & Os10g0462900 & $\begin{array}{l}\text { Chaperonin CPN60-1, } \\
\text { mitochondrial }\end{array}$ & 2.1 & 0.03 & 6 & EGVITIADGNTLYNELEVVEGMK \\
\hline & & & & & & & & EGVITIADGNTLYNELEVVEGMKLDR \\
\hline & & & & & & & & LLEQDNTDLGYDAAK \\
\hline & & & & & & & & SVAAGMNAMDLR \\
\hline & & & & & & & & TALVDAASVSSLMTTTESIIVEIPKEEK \\
\hline & & & & & & & & VTVSKDDTVILDGAGDKK \\
\hline & 10 & Q6ZFJ9 & Os02g0102900 & $60 \mathrm{kDa}$ chaperonin beta subunit & 2.0 & 0.04 & 2 & EVELEDPVENIGAK \\
\hline & & & & & & & & LADLVGVTLGPK \\
\hline & 13 & Q8H903 & Os10g0462900 & $60 \mathrm{kDa}$ chaperonin, mitochondrial & 2.3 & 0.01 & 2 & DGNTLYNELEVVEGMKLDRGYISPYFVTNPK \\
\hline
\end{tabular}


Table 1 (continued)

\begin{tabular}{|c|c|c|c|c|c|c|c|c|}
\hline Function & Spot no. & Uniprot no. & Oryza sativa no. & Annotation & $\mathrm{FC}^{\mathrm{a}}$ & P-value & Peptides $^{\mathrm{b}}$ & Peptide sequences \\
\hline \multirow[t]{8}{*}{ Signaling } & 1 & Q7EYF8 & Os07g0145400 & Protein kinase & 2.1 & 0.03 & 2 & GGLVVSADELGAPR \\
\hline & & & & & & & & RGPPLTWAQRLKIAVDVARGLNY \\
\hline & 4 & Q7FAZ2 & Os04g0202300 & $\begin{array}{l}\text { Lectin S-receptor-like serine/ } \\
\text { threonine protein kinase LecRK2 }\end{array}$ & 2.2 & 0.05 & 2 & DPSGNEVWNPRVTDVGYARMLDTGNFR \\
\hline & & & & & & & & SPSMISSGSSKWKKDKKYWILGSSLFFGSSVLVN \\
\hline & 9 & Q6ZF83 & Os01g0889900 & Serine/threonine protein kinase & 2.8 & 0.07 & 2 & TTGVSQRLVPWRNNANPSPGLFSLELD \\
\hline & & & & & & & & ALIILAIVLFIVFQKCRRDRTLR \\
\hline & 12 & Q6H7I7 & Os02g0634700 & Serine carboxypeptidase II & 2.7 & 0.05 & 2 & FPQYKSHDFYIAGESYAGHYVPQLSEK \\
\hline & & & & & & & & NWTHCSDVIGKWRDAPFSTLPIIRKLVAGGI \\
\hline
\end{tabular}

${ }^{\text {a }}$ Fold change; positive and negative FC values indicate elevated and reduced levels, respectively, in MHR4 as compared to HS1 Avena fatua plants.

${ }^{b}$ Number of uniquely matched peptides.

processes, xenobiotic and stress response, ROS detoxification and redox maintenance, chaperones and heat shock proteins, and signaling proteins. Overall, signals from the majority of proteins with functions in core cellular processes, photosynthesis, and translation were constitutively lower in MHR4 than HS1 plants, while those involved in xenobiotic response, ROS metabolism, redox maintenance, heat shock response, and signaling were constitutively elevated in MHR4 plants.

\subsection{Core cellular processes}

Differential signals from six of nine phosphorylated proteins involved in basic metabolism were constitutively reduced in MHR4 plants (Table 1), including hexokinase, fructose-bisphosphate aldolase, sedoheptulose-1,7-bisphosphatase, granule-bound starch synthase I, phosphoribulokinase, and 2-dehydro-3-deoxyphosphooctonate aldolase (spots 16-21, Fig. 1). These enzymes are involved in the biosynthesis or sensing of primary metabolites, specifically glycolysis, starch synthesis, lipopolysaccharide biosynthesis, the Calvin cycle, and glucose sensing. In contrast, glutamine synthetase, ATP synthase CF1 beta subunit, and transketolase (spots 6, 7, and 14, Fig. 1) were constitutively elevated in MHR4 plants. Phosphorylated glutamine synthetase, transketolase, and fructose-bisphosphate aldolase were identified in Arabidopsis thatiana and $O$. sativa in response to pathogen attack (Jones et al., 2006) and abscisic acid application (He and Li, 2008), respectively.

Differential signals of redox-sensitive proteins involved in photosynthesis were mixed, in that photosystem I reaction center subunit II (spot 41 ) and $23 \mathrm{kDa}$ polypeptide of photosystem II (spot 39) were reduced and elevated in MHR4 plants, respectively (Fig. 2, Table 2). Photosystem I and II reaction centers are a primary site of ROS generation (Asada, 2006), and a redox sensitive photosystem I light harvesting complex protein was identified in response to methyl jasmonate treatment in A. thaliana (Alvarez et al., 2009).

Signals of four redox-sensitive proteins involved in protein translation were differentially reduced in MHR4 plants, including chloroplast translational elongation factor Tu, plastid-specific $30 \mathrm{~S}$ ribosomal protein 2, and 40S ribosomal protein S14 (spots 40, 45, and 47, respectively) (Fig. 2, Table 2). In contrast, elongation factor 1-beta 1 (spot 31, Fig. 2) was elevated in MHR4 as compared to HS1 plants. Ribosomal and elongation factor proteins were redox-sensitive in A. thaliana (Wang et al., 2011), while the Tu elongation factor was induced by stress treatments in E. coli (Leichert et al., 2008).

Signals from other phosphorylated proteins identified at reduced constitutive levels in MHR4 plants include a putative polyprotein, poly (ADP-ribose) polymerase, a NAD(P)-binding domain containing protein, and aminomethyltransferase (spots 15, 22-24) (Fig. 1, Table 1). Of these, the putative aminomethyltransferase is of interest since this enzyme was also identified at lower levels in salt-stressed Beta vulgaris (Wakeel et al., 2011) and was phosphorylated in response to pathogen attack in A. thaliana (Jones et al., 2006).

These overall reductions in phosphorylation and redox signals from core cellular proteins in MHR4 plants may represent an energetic tradeoff as predicted by the resource-based allocation theory (Coley et al.,

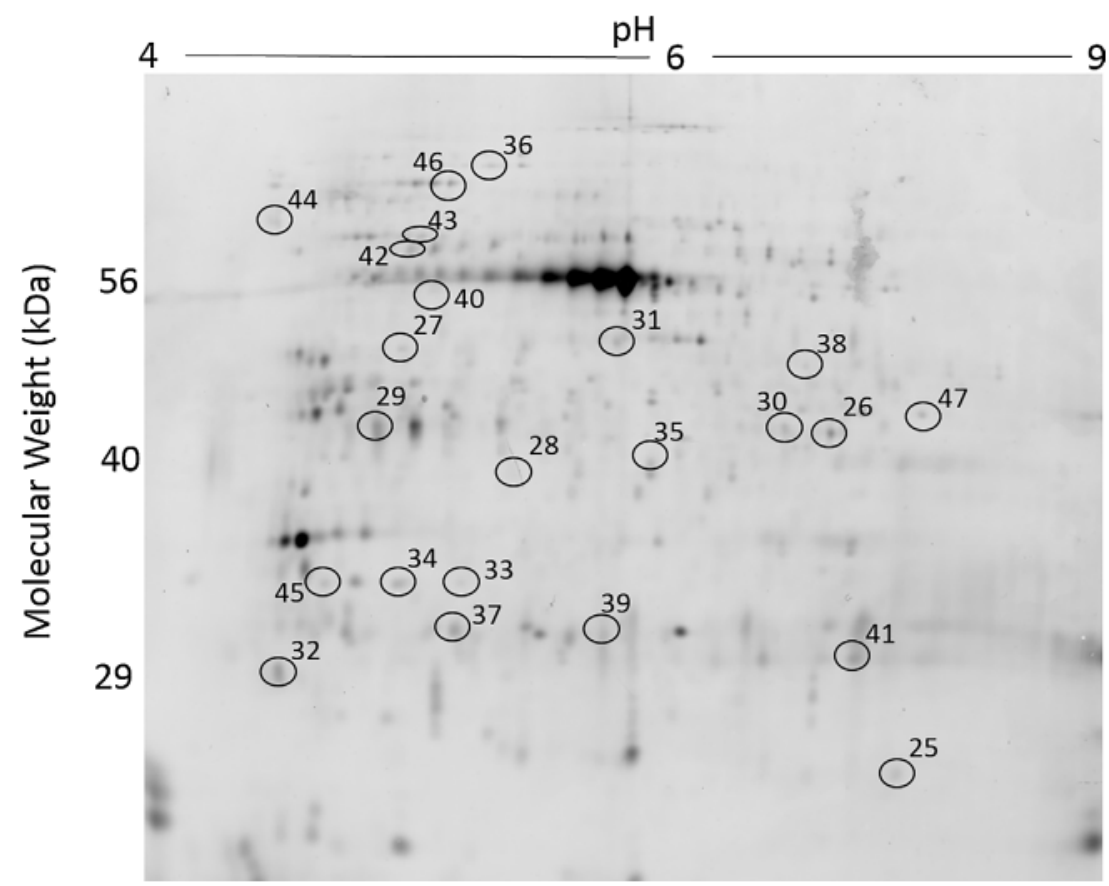

Fig. 2. Representative two dimensional electrophoresis gel of the multiple herbicide resistant (MHR4) Avena fatua redox proteome. Significantly differential (between MHR and herbicide susceptible (HS1) plants) protein spots were numbered, excised from gels, and identified by LC MS/MS. Spot numbers are the same as in Table 2. 
Table 2

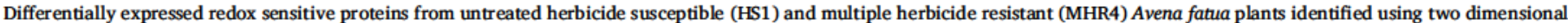
polyacrylamide gel electrophoresis.

\begin{tabular}{|c|c|c|c|c|c|c|c|c|}
\hline Function & Spot no. & Uniprot no. & Oryza sativa no. & Annotation & $\mathrm{FC}^{\mathrm{a}}$ & P-value & Peptides $^{b}$ & Peptide sequence \\
\hline \multirow[t]{17}{*}{ Core cellular processes } & 31 & Q40680 & Os07g0614500 & Elongation factor 1-delta 1 & 1.4 & 0.10 & 2 & $\begin{array}{l}\text { NVKMEGLLWGASK } \\
\text { AAEERAAAVKASGK }\end{array}$ \\
\hline & 39 & BOFFP0 & Os07g0141400 & $23 \mathrm{kDa}$ polypeptide of photosystem II & 1.5 & 0.08 & 2 & $\begin{array}{l}\text { AANVFGKPKTNTEF } \\
\text { HQLITATVNDGKLYICKAQAGD }\end{array}$ \\
\hline & 40 & I1 IB68 & Os02g0595700 & $\begin{array}{l}\text { Chloroplast translational elongation } \\
\text { factor Tu }\end{array}$ & -1.4 & 0.00 & 6 & GITINTATVEYETETR \\
\hline & & & & & & & & NATVTGVEMFQK \\
\hline & & & & & & & & TMDDAIAGDNVGLLLR \\
\hline & & & & & & & & TTDVTGNVTNIMNDKDEEAK \\
\hline & & & & & & & & VGDPVDLVGIR \\
\hline & & & & & & & & VGDPVDLVGIRETR \\
\hline & 41 & Q84PB4 & Os08g0560900 & $\begin{array}{l}\text { Photosystem I reaction center subunit } \\
\text { II }\end{array}$ & -2.3 & 0.06 & 3 & EQVFEMPTGGAAIMR \\
\hline & & & & & & & & FTGKNTFDV \\
\hline & & & & & & & & ARKEQCLALGTRLRSKYKINYQ \\
\hline & 45 & Q6H443 & Os09g0279500 & $\begin{array}{l}\text { Plastid-specific } 30 \text { S ribosomal protein } \\
2\end{array}$ & -2.1 & 0.10 & 2 & SRLTVGAARWWARRRQPAVVVR \\
\hline & & & & & & & & AARKLYVGNIPRTVT \\
\hline & 47 & B1NEV4 & Os02g0534800 & $40 S$ ribosomal protein $S 14$ & -3.0 & 0.10 & 4 & KRGKVQKEEVQ \\
\hline & & & & & & & & HIFASFNDTFVHVTDLS \\
\hline & & & & & & & & MLAAQDVAEKCKSLGI \\
\hline & & & & & & & & RATGGNKTKTPGPGAQSALRA \\
\hline \multirow{15}{*}{$\begin{array}{l}\text { Xenobiotic and stress } \\
\text { response }\end{array}$} & 28 & І1ІІІ3 & Os11g0145200 & Anthocyanin 5-0-glucosyltransferase & 5.2 & 0.07 & 2 & DFPTFLVDTTGSDIASSVNEALR \\
\hline & & & & & & & & VLAAYYHFFHDDGGHYK \\
\hline & 29 & Q10A56 & Os10g0140200 & Glycoside hydrolase & 1.3 & 0.10 & 4 & GVGEPLNEVVCVDQKCDGLVAR \\
\hline & & & & & & & & AEQAFFQRWWAEKSPKIQAIV \\
\hline & & & & & & & & RNMDRLINYVNKDGRVHALYSTP \\
\hline & & & & & & & & EVEYTIGPIPVDDDDDIGK \\
\hline & 32 & Q0JG98 & Os01g0934900 & $\begin{array}{l}\text { Esterase PIR7A, putative } \\
\text { carboxylesterase }\end{array}$ & 1.5 & 0.05 & 3 & FPDKVAAAVFLAACMPAAGK \\
\hline & & & & & & & & AAAGAHPARADEVGSLEE \\
\hline & & & & & & & & EGNYGSVKRVFLVAMDDASSDE \\
\hline & 34 & Q8LMC7 & Os07g0267400 & Ulp1 protease & 1.6 & 0.08 & 3 & RFHFPCAKQDQR \\
\hline & & & & & & & & YNTEFHWVLLFFD \\
\hline & & & & & & & & YSTLSKTPCLYGSTPRSTKA \\
\hline & 37 & Q2QS17 & Os12g0443000 & Cytochrome P450 family protein & 1.4 & 0.05 & 3 & AFTDVLGDLLGGGIFNADGERWFAQRK \\
\hline & & & & & & & & QLLAAARGRDDLVSRM \\
\hline & & & & & & & & RMEAIWGADAGEFRPGRWLAAAA \\
\hline \multirow{11}{*}{$\begin{array}{l}\text { ROS and redox } \\
\text { maintenance }\end{array}$} & 26 & I1 HB66 & Os01g0107900 & DUF3506 domain containing protein & 2.7 & 0.10 & 2 & GNEDTEEKTQDVGNTK \\
\hline & & & & & & & & VKLFISGVVHNKEDMAGAKS \\
\hline & 30 & Q942J6 & Os07g0119400 & Putative L-ascorbate oxidase & 1.3 & 0.04 & 2 & AEDPYHFFDWK \\
\hline & & & & & & & & TRTIMDVAQKVMLINDMFPGPTI \\
\hline & 33 & Q5U1J1 & Os07g0499500 & Peroxidase 7 & 1.5 & 0.05 & 2 & $\begin{array}{l}\text { LFFHDFAVQGIDASVLVDSPGSERYAK } \\
\text { YWPLMYGRKDGRRSSMVDA }\end{array}$ \\
\hline & 35 & Q8W3D0 & Os10g0100700 & $\begin{array}{l}\text { Putative pyridoxal 5'-phosphate } \\
\text { synthase subunit PDX1.2 }\end{array}$ & 2.4 & 0.08 & 2 & AIVQAVTHYSDPK \\
\hline & & & & & & & & EVSSGLGEAMVGINLSD \\
\hline & 36 & B7ERQ1 & Os07g0638300 & 1-Cys peroxiredoxin & 1.5 & 0.07 & 2 & QLNMVDPDEK \\
\hline & & & & & & & & KLLGISCDDVQSHKDWIKDIEAYKP \\
\hline & 38 & Q6EQV9 & Os02g0320800 & $\begin{array}{l}\text { Iron/ascorbate-dependent } \\
\text { oxidoreductase }\end{array}$ & 2.2 & 0.10 & 2 & DVLRAMARIAGLDDDDQHFVDQLG \\
\hline & & & & & & & & RFNYYPPCPRPDLVMGIKPHSDG \\
\hline \multirow{8}{*}{$\begin{array}{l}\text { Chaperones and heat } \\
\text { shock }\end{array}$} & 25 & C6F1N7 & Os03g0804800 & CCt8 protein like & 1.9 & 0.10 & 2 & TSLGPNGMNKMVINHLDK \\
\hline & & & & & & & & YAIAKFAESFEMVPRTLAENAGLSAMEV \\
\hline & 42 & Q9LWT6 & Os06g0114000 & $60 \mathrm{kDa}$ chaperonin & -2.3 & 0.04 & 3 & EVELEDPVENIGAK \\
\hline & & & & & & & & $\begin{array}{l}\text { IVNDGVTVAREVELEDPVENIGAK } \\
\text { LAGGVAVIOVGAOTETELKEK }\end{array}$ \\
\hline & 43 & Q6ZFJ9 & Os02g0102900 & $60 \mathrm{kDa}$ chaperonin beta & -1.2 & 0.10 & 2 & EVELEDPVENIGAK \\
\hline & & & & & & & & LAGGVAVIQVGAQTETELKEK \\
\hline & 44 & Q9LGR0 & Os01g0184900 & FACT complex subunit SSRP1-A & -1.6 & 0.01 & 2 & TDGHLFNNILLGGRAGSNPGQFK \\
\hline & & & & & & & & SPTDDSGGEDSDASESGGEKEKLSKKEA \\
\hline \multirow[t]{5}{*}{ Signaling } & 27 & Q67WN0 & Os06g0644466 & L-zip + NBS + LRR-like protein & 1.3 & 0.10 & 3 & SNFIEDSSMAEDK \\
\hline & & & & & & & & IGSSNLALIALKINDSGSSSDIV \\
\hline & & & & & & & & WKSIPHLELLNITELTIDKCVDSCPVPK \\
\hline & 46 & Q69Q47 & Os06g0606000 & $\begin{array}{l}\text { CBL-interacting serine/threonine- } \\
\text { protein kinase } 24\end{array}$ & -1.4 & 0.10 & 2 & YFQQLIDAINYCHSKGVYHR \\
\hline & & & & & & & & SPFAVVLQVFEVAPSLFMVDVR \\
\hline
\end{tabular}

${ }^{\text {a }}$ Fold change; positive and negative FC values indicate elevated and reduced levels, respectively, in MHR4 as compared to HS1 Avena fatua plants.

${ }^{b}$ Number of uniquely matched peptides. 
1985). Briefly, heritable resistance to an environmental stress or SAA may require the reallocation of carbon away from core processes to stress-related pathways, resulting in a new homeostasis (Kosová et al., 2011). More specifically, PTMs are often employed to modulate enzyme activities under stress conditions because they confer immediate and selective changes (Holcik and Sonenberg, 2005). The physiological result of these changes can be manifested in fitness costs and a resulting ecological disadvantage (Bazzaz et al., 1987), both of which are documented for SAR (Bergelson and Purrington, 1996), SAA (Zhen et al., 2011), and herbicide resistance (Vila-Aiub et al., 2009). For $A$. fatua, our previous greenhouse experiments showing that MHR plants produced fewer tillers and seeds than HS plants (Lehnhoff et al., 2013) are consistent with the idea that reduced activities of core cellular processes can be reflected in plant growth reductions.

\subsection{Xenobiotic and stress response}

Signals of five redox-sensitive proteins, anthocyanin 5-O-glucosyltransferase, cytochrome P450 monooxygenase, carboxylesterase, Ulp1 protease, and glycoside hydrolase (spots $28,37,32,34$, and 29 , respectively, Fig. 2), were constitutively elevated in MHR4 as compared to HS1 plants (Table 2). The large family of plant glycosyltransferases has roles in Phase II herbicide metabolism (Yuan et al., 2007) as well as conferring tolerance to abiotic stresses like salt, cold, and drought by modifying anthocyanin accumulation (Li et al., 2017). We recently reported on a closely related anthocyanidin 3-O-glucosyltransferase differentially expressed gene (DEG) as one of four glucosyltransferase DEGs constitutively elevated in MHR4 plants, and differential expression of three of these co-segregated with flucarbazone-sodium herbicide resistance in $F_{3}$ families (Keith et al., 2017). Similarly, P450s are wellknown participants in Phase I xenobiotic and herbicide metabolism (Yuan et al., 2007). Our transcriptome study (Keith et al., 2017) identified two P450s elevated in MHR4 plants, and differential expression levels of one co-segregated with herbicide resistance in $\mathrm{F}_{3}$ families. Higher constitutive levels of an oxidized carboxylesterase in MHR4 plants may be related to differential de-esterification of pro-herbicides like fenoxaprop-P-ethyl and imazamethabenz-methyl to their respective toxic carboxylic acids (Cummins et al., 2001). Elevated levels of Ulp1 proteases, regulators of protein sumoylation (Novatchkova et al., 2004) are documented after plant pathogen attack (Hanania et al., 1999) and in abiotic stress response (Kurepa et al., 2003). And finally, glycoside hydrolases, enzymes with functions in polysaccharide metabolism, are involved in plant defense against pathogens (Minic, 2008).

\subsection{ROS detoxification and redox maintenance}

Abiotic stresses including herbicides cause rapid ROS generation and thus disrupt redox homeostasis (Dayan and Watson, 2011; Demidchik, 2015; Dietz, 2014). Given this relationship, MHR4 plants should exhibit enhanced capacity for ROS management, given that they are resistant to known ROS-producing herbicides and families like paraquat (Dodge, 1971), difenzoquat (Kovacic and Somanathan, 2014), ALS inhibitors (Zulet et al., 2015), and ACCase inhibitors (Luo et al., 2004). In this regard, two phosphorylated peroxiredoxins (spots 2 and 11, Fig. 1), thioredoxin-dependent peroxidases that reduce $\mathrm{H}_{2} \mathrm{O}_{2}$ and organic peroxides, were constitutively elevated in MHR4 plants (Table 1). Increased levels of these enzymes should improve the capacity for ROS degradation (Muthuramalingam et al., 2009) in MHR plants. Similarly, redox-sensitive spots 33 and 36 (Fig. 2), annotated as a peroxidase and 1-Cys peroxiredoxin, respectively, were constitutively elevated in MHR4 plants (Table 2). Both enzymes were elevated in A. thaliana following $\mathrm{H}_{2} \mathrm{O}_{2}$ treatment (Wang et al., 2011). We also identified a peroxiredoxin DEG that was constitutively elevated in MHR plants in our transcriptome study (Keith et al., 2017).

Nearly half of the redox-sensitive proteins identified in this study have roles in redox maintenance, with the majority of them elevated in
MHR4 plants (Table 2). Two proteins, L-ascorbate oxidase and iron/ ascorbate-dependent oxidoreductase (spots 30 and 38, Fig. 2) are involved in ascorbate metabolism, and thus play a significant role in defense against oxidative stress (Smirnoff, 2000). We recently demonstrated that the specific activity of dehydroascorbate reductase, a key enzyme of the glutathione-ascorbate ROS protective pathway (Asada, 2006), was 1.4-fold higher in MHR4 versus HS1 plants (Burns et al., 2017). Together, these results indicate that ascorbic acid-mediated ROS activity is constitutively elevated in MHR plants, with the potential to ameliorate the damaging oxidative effects of herbicides.

Signals of two redox-sensitive proteins with roles in singlet oxygen metabolism were constitutively elevated in MHR4 plants (Table 2). Spot 26 (Fig. 2), annotated as a DUF3506 domain-containing protein, shares strong similarity with the EX1 and EX2 proteins from A. thaliana proteins that detoxify light-stress generated singlet oxygen (Wagner et al., 2004). Spot 35 (Fig. 2) was annotated as pyridoxal 5'-phosphate synthase subunit PDX1.2, an enzyme of vitamin B6 biosynthesis that indirectly contributes to ROS tolerance (Titiz et al., 2006).

To investigate the potential role of superoxide dismutase (SOD) in ROS metabolism (Gill et al., 2015) potentially related to NTSR, we compared constitutive enzyme activities and protein levels in MHR and HS plants. SOD enzyme activities as determined in native gels showed one major band and diffuse minor bands of activity, with no detectable differences between HS and MHR plants (Fig. 3a). Polyclonal antibodies against chloroplastic and cytosolic CuZn SODs (Kanematsu and Asada, 1990) were subsequently used in immunoblots to compare levels of

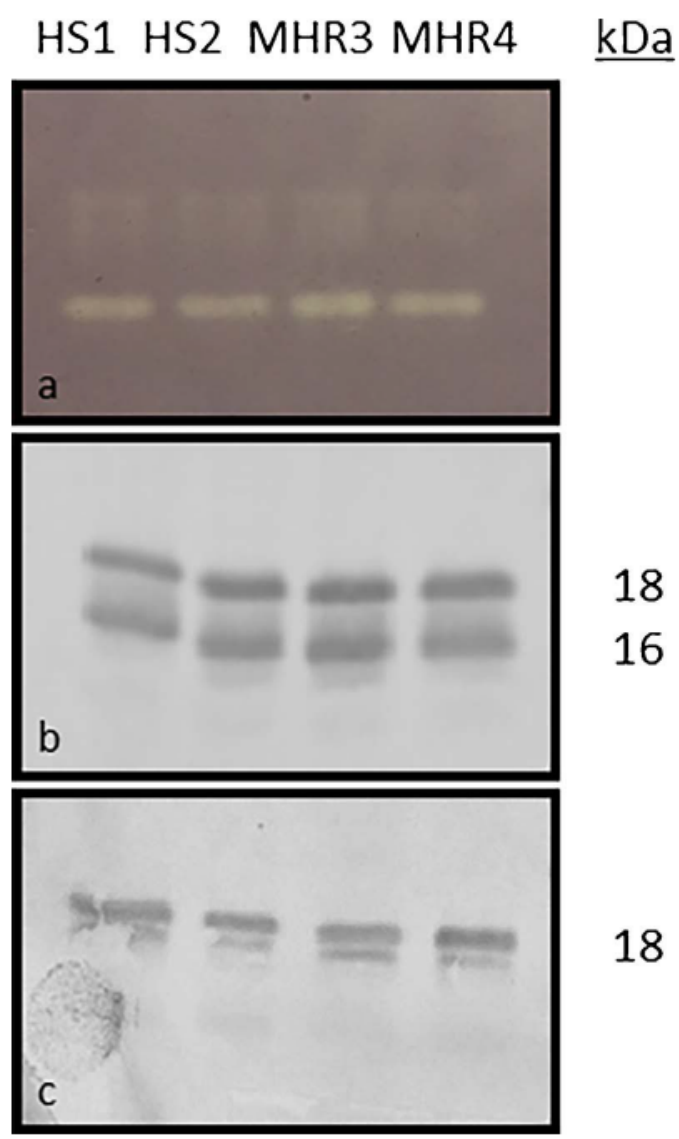

Fig. 3. (a) Superoxide dismutase activity native gel of extracts from untreated herbicide susceptible (HS1 and HS2) and multiple herbicide resistant (MHR3 and MHR4) Avena fatua plants. (b) 1D immunoblot of proteins from untreated herbicide susceptible (HS1 and HS2) and multiple herbicide resistant (MHR3 and MHR4) Avena fatua plants recognized by anti-chloroplastic CuZn superoxide dismutase antibodies. (c) $1 \mathrm{D}$ immunoblot of proteins from untreated herbicide susceptible (HS1 and HS2) and multiple herbicide resistant (MHR3 and MHR4) Avena fatua plants recognized by anti-cytosolic CuZn superoxide dismutase antibodies. 
immunoreactive proteins in MHR and HS plants (Fig. 3b-c). These antibodies have been successfully used to identify cytosolic and chloroplastic CuZn SODs in S. oleracea, O. sativa, and Equisetum arvense (Kanematsu and Asada, 1990). Both antibodies recognized two proteins of approximately 16 and $18 \mathrm{kDa}$ in extracts from $A$. fatua plants (Fig. 3b-c), and densitometric scans confirmed that constitutive protein amounts were not different among plants (data not shown). This lack of difference in chloroplastic and cytosolic CuZn SOD enzyme activities and amounts between MHR and HS plants may indicate that other SODs or ROS management pathways like the ascorbate-related changes noted above are sufficient to prevent or ameliorate herbicide-induced ROS damage. In this regard, we previously identified a chloroplastic Fe SOD DEG at constitutively elevated levels in MHR4 as compared to HS1 plants (Keith et al., 2017).

\subsection{Chaperones and heat shock proteins}

Six protein spots were annotated with chaperone activity: five phosphorylated heat shock proteins including Hsp60 (spots 5, 8, 10, and 13; Fig. 1) and Hsp70 (spot 3; Fig. 1), and a single redox-sensitive protein annotated as a CCt8 protein (spot 25; Fig. 2). Signals from all proteins were constitutively elevated in MHR4 plants (Tables 1 and 2). Hsps have well-known roles as molecular chaperones that prevent protein misfolding, regulation of transcription factors (TFs) for a diverse set of genes (Morimoto, 2002), and are themselves regulated by phosphorylation (Muller et al., 2013). Higher constitutive amounts or phosphorylation status of Hsps in MHR plants may allow them to better protect key proteins during herbicide-mediated stress. Although information about phosphorylated Hsps in plants is sparse, phosphorylated Hsps were shown to protect against oxidative stress in humans (Kalmar and Greensmith, 2009) and Trichinella spiralis (Martinez et al., 2002).

In contrast to the above proteins, signals from two redox-sensitive Hsp60 proteins (spots 42 and 43; Fig. 2) and a FACT complex subunit SSRP1-A (spot 44; Fig. 2) were constitutively reduced in MHR4 plants (Table 2). FACT complexes act as histone chaperones during transcription elongation and were reduced by salt stress in rice (Pandit et al., 2011).

\subsection{Signaling}

Signals from five proteins involved in cell signaling were constitutively elevated in MHR4 plants (Tables 1 and 2), including phosphorylated serine carboxypeptidase II (spot 12; Fig. 1), redox-sensitive nucleotide-binding site leucine-rich repeat (NBS-LRR) protein (spot 27; Fig. 2), and three phosphorylated protein kinases (spots 1, 4, and 9; Fig. 1). In addition to their roles in storage protein turnover, serine carboxypeptidases can be involved in brassinosteroid and receptor-like kinase signaling (Zhou and Li, 2005). NBS-LRR proteins constitute the majority of disease resistance genes in plants, confer resistance to a diverse array of pathogens (Marone et al., 2013), provide enhanced drought and salt tolerance (Xinlong et al., 2016), and play key roles in plant defense responses (Belkhadir et al., 2004). Our transcriptome study similarly identified an NBS-LRR DEG that was constitutively elevated in MHR4 plants and its differential expression co-segregated with herbicide resistance in $\mathrm{F}_{3}$ families (Keith et al., 2017). In contrast, a redox-sensitive CBL-interacting serine/threonine-protein kinase (spot 46; Fig. 2) was constitutively reduced in MHR4 plants (Table 2). The $O$. sativa ortholog of this kinase was shown to phosphorylate and thus activate a plasma membrane $\mathrm{Na}^{+} / \mathrm{H}^{+}$antiporter involved in salt tolerance (Martínez-Atienza et al., 2007).

Phosphorylated spots 1, 4, and 9 (Fig. 1), annotated as protein kinases, were constitutively elevated in MHR4 plants (Table 1). Specifically spot 4 was annotated as the lectin S-receptor-like serine/threonine-protein kinase LecRK2, a well-characterized receptor in SAR and PTI (Boller and Felix, 2009). PTI is initiated by perception of microbial, pathogen, or damage-associated patterns by cell surface-localized receptor-like proteins or RLKs, some of which possess an extracellular lectin motif (LecRKs) and an intracellular kinase domain (Singh and Zimmerli, 2013). In general, the linkage between RLK ligand perception and signaling initiation/specificity is tightly regulated by the state of RLK phosphorylation (Macho and Zipfel, 2014), and the activities of certain LecRKs are enhanced by phosphorylation (Nishiguchi et al., 2002; Vaid et al., 2016).

PTI and SAA can be 'primed' by initial plant exposure to pathogens, abiotic stress, or disparate chemicals (Pastor et al., 2013), and primed plants exhibit enhanced and durable resistance to pathogens and subsequent abiotic stresses (Savvides et al., 2016; Zimmerli et al., 2008). Priming is thought to involve the accumulation of inactive protein kinases, TFs, or other defense signaling components, that are rapidly activated upon stress (Pastor et al., 2014). For example, MAPK3 and MAPK6 in A. thaliana were strongly activated in plants primed by exposure to biotic and abiotic stresses (Beckers et al., 2009). LecRK2 and two additional LecRK genes were shown to confer broad-spectrum and durable resistance against insects pests in rice (Liu et al., 2015). In MHR4 A. fatua, the elevation and/or differential phosphorylation of LecRK2 and two other protein kinases confirm that MHR4 plants exhibit constitutive changes in known SAR- and SAA-related proteins. If in fact these changes are associated with 'priming' the MHR4 phenotype, LecRK2 may be binding to the herbicide itself or features induced by herbicide entry. Similar activation has been documented in response to diverse compounds like salt (Deng et al., 2009), allyl-isothiocyanate (Kissen et al., 2016), and extracellular ATP (Cao et al., 2014) for related L-type LecRKs.

\section{Conclusion}

This work seeks to better understand the molecular mechanisms of NTSR in MHR A. fatua by surveying differential signals from phosphorylated and redox-sensitive proteins. Our results demonstrate that PTMs of a number of proteins with functions in core cellular processes, xenobiotic and stress response, ROS detoxification and redox maintenance, chaperones/heat shock response, and intracellular signaling are constitutively altered in MHR4 as compared to HS1 plants. Clearly, additional work will be required before causal relationships can be assigned between these proteins and NTSR. Using a candidate gene approach, individual proteins are being pursued biochemically, and the rudimentary mapping populations we have developed (Burns et al., in press) provide the basis for additional genetic or QTL-based strategies, with the aid of the recently published Avena sativa linkage map (Chaffin et al., 2016).

Regardless of their potential roles in NTSR, the protein functions described here are shared with plant biotic (SAR) and abiotic (SAA) stress response pathways. For example, elevated stress responses, enhanced ROS detoxification, alterations in protein kinases, and fitness penalties (as a result of implied reduced biosynthetic activities) are well documented in SAR and SAA. The MHR A. fatua PTMs related to ROS detoxification, LecRK2, and other protein kinases are especially relevant, since these features play fundamental roles in the cross-talk between plant biotic and abiotic stress responses (Atkinson and Urwin, 2012; Rejeb et al., 2014). More specifically, MAP kinase cascades described for SAA and SAR are known to translate ROS signals into PTMs like protein phosphorylation and oxidation, resulting in signal transduction modulations needed for stress perception and response (Dietz et al., 2016; Kosová et al., 2011; Waszczak et al., 2015). The specific protein PTMs reported here for MHR A. fatua are fully consistent with this model.

Priming of PTI can require multiple, consecutive exposures to modest stresses (Boller and Felix, 2009), as has been noted for the evolution of NTSR (Délye et al., 2013; Neve and Powles, 2005). For MHR A. fatua, more than 35 years of annual herbicide applications were required before this phenotype was detected (Keith et al., 2015). 
Different herbicide modes of action were applied consecutively but rarely in mixtures in the fields where MHR A. fatua evolved (Keith et al., 2015), demonstrating that the longstanding resistance-prevention recommendation of rotating herbicides (Shaner, 2014) was ineffective under these conditions. The similarities between herbicide resistance and acclimation (Vila-Aiub and Ghersa, 2005) or SAR (Molina et al., 1999) have received some attention (Délye et al., 2013; Dubey et al., 2016; Perez and Brown, 2014), although specific evidence connecting the two is scant. We suggest that the constitutive changes documented here further support the idea (Alberto et al., 2016) that herbicide stress is perceived by plants in the same fashion as other abiotic stresses, and that NTSR shares a number of similar features with SAR and SAA. Further, we speculate that MHR A. fatua's previous exposure to sublethal herbicide doses, as well as earlier evolution under a diversity of abiotic and biotic stressors has led to a heightened state of stress preparedness that includes NTSR to a number of dissimilar herbicides. Further investigations into the evolution of these related responses through the lens of phenotypic convergence (Baucom, 2016; Losos, 2011) may yield valuable insights into the commonalities among plant responses to selection by biotic, abiotic, and herbicide stressors.

\section{Acknowledgements}

Polyclonal antisera to the spinach chloroplastic and cytosolic CuZn SODs (Kanematsu and Asada, 1990) were graciously provided by Dr. Sumio Kanematsu, Minami-Kyushu University, Japan.

The excellent technical assistance of Tara Donohoe, Alex Griffin, and Katie Steward is much appreciated. This work was partially supported by USDA-NIFA-AFRI grants 2012-67013-19467 and 201667013-24888, US EPA Strategic Agricultural Initiative grant X897873401-0, Bayer CropScience, the Montana Noxious Weed Trust Fund, the Montana Wheat and Barley Committee, and the Montana Agricultural Experiment Station. The proteomics, metabolomics, and mass spectrometry facility at MSU receives support from the Murdock Charitable Trust and Montana INBRE under Award Number P20GM103474 from the National Institutes of Health (NIGMS).

\section{References}

Agrawal, G.K., Thelen, J.J., 2005. Development of a simplified, economical polyacrylamide gel staining protocol for phosphoproteins. Proteomics 5 (18), 4684-4688.

Alberto, D., Serra, A.-A., Sulmon, C., Gouesbet, G., Couée, I., 2016. Herbicide-related signaling in plants reveals novel insights for herbicide use strategies, environmental risk assessment and global change assessment challenges. Sci. Total Environ. 569, 1618-1628.

Alvarez, S., Zhu, M., Chen, S., 2009. Proteomics of Arabidopsis redox proteins in response to methyl jasmonate. J. Proteomics 73 (1), 30-40.

Asada, K., 2006. Production and scavenging of reactive oxygen species in chloroplasts and their functions. Plant Physiol. 141 (2), 391-396.

Atkinson, N.J., Urwin, P.E., 2012. The interaction of plant biotic and abiotic stresses: from genes to the field. J. Exp. Bot. 63 (10), 3523-3543.

Baucom, R.S., 2016. The remarkable repeated evolution of herbicide resistance. Am. J. Bot. 103 (2), 181-183.

Bazzaz, F.A., Chiariello, N.R., Coley, P.D., Pitelka, L.F., 1987. Allocating resources to reproduction and defense. Bioscience 37 (1), 58-67.

Beckers, G.J., Jaskiewicz, M., Liu, Y., Underwood, W.R., He, S.Y., Zhang, S., Conrath, U., 2009. Mitogen-activated protein kinases 3 and 6 are required for full priming of stress responses in Arabidopsis thaliana. Plant Cell 21 (3), 944-953.

Belkhadir, Y., Subramaniam, R., Dangl, J.L., 2004. Plant disease resistance protein signaling: NBS-LRR proteins and their partners. Curr. Opin. Plant Biol. 7 (4), 391-399.

Bergelson, J., Purrington, C.B., 1996. Surveying patterns in the cost of resistance in plants. Am. Nat. 148 (3), 536-558.

Boller, T., Felix, G., 2009. A renaissance of elicitors: perception of microbe-associated molecular patterns and danger signals by pattern-recognition receptors. Annu. Rev. Plant Biol. 60, 379-406.

Bradford, M.M., 1976. A rapid and sensitive method for the quantitation of microgram quantities of protein utilizing the principle of protein-dye binding. Anal. Biochem. 72 (1-2), 248-254.

Burns, E.E., Keith, B.K., Refai, M.Y., Bothner, B., Dyer, W.E., 2017. Proteomic and biochemical assays of glutathione-related proteins in susceptible and multiple herbicide resistant Avena fatua L. Pestic. Biochem. Physiol. 140, 69-78.

Burns E.E., Keith B.K., Talbert L.E., Dyer W.E. Non-target site resistance to flucarbazone, imazamethabenz and pinoxaden is controlled by three linked genes in Avena fatua. Weed Research https://doi.org/10.1111/wre.12279.
Cao, Y., Tanaka, K., Nguyen, C.T., Stacey, G., 2014. Extracellular ATP is a central signaling molecule in plant stress responses. Curr. Opin. Plant Biol. 20, 82-87.

Caseley, J., Walker, A., 1990. Entry and transport of herbicides in plants. Entry and Transport of Herbicides in Plant, 8 ed. pp. 183-215.

Chaffin, A.S., Huang, Y.-F., Smith, S., Bekele, W.A., Babiker, E., Gnanesh, B.N., Foresman, B.J., Blanchard, S.G., Jay, J.J., Reid, R.W., 2016. A consensus map in cultivated hexaploid oat reveals conserved grass synteny with substantial subgenome rearrangement. Plant Genome 9 (2).

Chen, C.-N., Pan, S.-M., 1996. Assay of superoxide dismutase activity by combining electrophoresis and densitometry. Bot. Bull. Acad. Sin. 37.

Cleveland, D.W., Fischer, S.G., Kirschner, M.W., Laemmli, U.K., 1977. Peptide mapping by limited proteolysis in sodium dodecyl sulfate and analysis by gel electrophoresis. J. Biol. Chem. 252 (3), 1102-1106.

Coley, P.D., Bryant, J.P., Chapin, F.S., 1985. Resource availability and plant antiherbivore defense. Science 230 (4728), 895-899.

Cummins, I., Burnet, M., Edwards, R., 2001. Biochemical characterisation of esterases active in hydrolysing xenobiotics in wheat and competing weeds. Physiol. Plant. 113 (4), 477-485.

Délye, C., Jasieniuk, M., Le Corre, V., 2013. Deciphering the evolution of herbicide resistance in weeds. Trends Genet. 29 (11), 649-658.

Délye, C., 2013. Unravelling the genetic bases of non-target-site-based resistance (NTSR) to herbicides: a major challenge for weed science in the forthcoming decade. Pest Manag. Sci. 69 (2), 176-187.

Das, M., Reichman, J.R., Haberer, G., Welzl, G., Aceituno, F.F., Mader, M.T., Watrud, L.S., Pfleeger, T.G., Gutiérrez, R.A., Schäffner, A.R., 2010. A composite transcriptional signature differentiates responses towards closely related herbicides in Arabidopsis thaliana and Brassica napus. Plant Mol. Biol. 72 (4-5), 545-556.

Dayan, F.E, Watson, S.B., 2011. Plant cell membrane as a marker for light-dependent and light-independent herbicide mechanisms of action. Pestic. Biochem. Physiol. 101 (3), 182-190.

Demidchik, V., 2015. Mechanisms of oxidative stress in plants: from classical chemistry to cell biology. Environ. Exp. Bot. 109, 212-228.

Deng, K., Wang, Q., Zeng, J., Guo, X., Zhao, X., Tang, D., Liu, X., 2009. A lectin receptor kinase positively regulates $\mathrm{ABA}$ response during seed germination and is involved in salt and osmotic stress response. J. Plant Biol. 52 (6), 493.

Dietz, K.J., Mittler, R., Noctor, G., 2016. Recent progress in understanding the role of reactive oxygen species in plant cell signaling. Plant Physiol. 171 (3), 1535-1539.

Dietz, K.J., 2014. Redox regulation of transcription factors in plant stress acclimation and development. Antioxid. Redox Signaling 21 (9), 1356-1372.

Dodds, P.N., Rathjen, J.P., 2010. Plant immunity: towards an integrated view of plant?pathogen interactions. Nat. Rev. Genet. 11 (8), 539-548.

Dodge, A., 1971. The mode of action of the bipyridylium herbicides paraquat and diquat. Endeavour 30, 130-135.

Dubey, G., Mishra, N., Prasad, S.M., 2016. Metabolic responses of pesticides in plants and their ameliorative processes. Plant Responses to Xenobiotics. Springer, pp. 57-95.

Dyballa, N., Metzger, S., 2009. Fast and sensitive colloidal coomassie G-250 staining for proteins in polyacrylamide gels. J. Visualized Exp. 30, e1431.

Fu, Z.Q., Dong, X., 2013. Systemic acquired resistance: turning local infection into global defense. Annu. Rev. Plant Biol. 64, 839-863.

Fujita, M., Fujita, Y., Noutoshi, Y., Takahashi, F., Narusaka, Y., Yamaguchi-Shinozaki, K., Shinozaki, K., 2006. Crosstalk between abiotic and biotic stress responses: a current view from the points of convergence in the stress signaling networks. Curr. Opin. Plant Biol. 9 (4), 436-442.

Gaines, T.A., Lorentz, L., Figge, A., Herrmann, J., Maiwald, F., Ott, M.C., Han, H., Busi, R., Yu, Q., Powles, S.B., 2014. RNA-Seq transcriptome analysis to identify genes involved in metabolism-based diclofop resistance in Lolium rigidum. Plant J. 78 (5), 865-876.

Georghiou, G.P., Taylor, C.E., 1986. Factors influencing the evolution of resistance. Pesticide Resistance: Strategies and Tactics for Management. pp. 157-169.

Gill, S.S., Anjum, N.A., Gill, R., Yadav, S., Hasanuzzaman, M., Fujita, M., Mishra, P., Sabat, S.C., Tuteja, N., 2015. Superoxide dismutase-mentor of abiotic stress tolerance in crop plants. Environ. Sci. Pollut. Res. 22 (14), 10375-10394.

Hanania, U., Furman-Matarasso, N., Ron, M., Avni, A., 1999. Isolation of a novel SUMO protein from tomato that suppresses EIX-induced cell death. Plant J. 19 (5), 533-541.

He, H., Li, J., 2008. Proteomic analysis of phosphoproteins regulated by abscisic acid in rice leaves. Biochem. Biophys. Res. Commun. 371 (4), 883-888.

Heap, I., 2014. Global perspective of herbicide-resistant weeds. Pest Manag. Sci. 70 (9), 1306-1315.

Hofer, M., Felsenstein, F., Petersen, M., 2014. Molecular analysis of metabolic resistance in blackgrass. Julius-Kühn-Archiv 443, 73-80.

Holcik, M., Sonenberg, N., 2005. Translational control in stress and apoptosis. Nat. Rev. Mol. Cell Biol. 6 (4), 318-327.

Jasieniuk, M., BrÛlé-Babel, A.L., Morrison, I.N., 1996. The evolution and genetics of herbicide resistance in weeds. Weed Sci. 176-193.

Johnson, R.R., Cranston, H.J., Chaverra, M.E., Dyer, W.E., 1995. Characterization of cDNA clones for differentially expressed genes in embryos of dormant and nondormant Avena fatua L, caryopses. Plant Mol. Biol. 28 (1), 113-122.

Jones, A.M., Bennett, M.H., Mansfield, J.W., Grant, M., 2006. Analysis of the defence phosphoproteome of Arabidopsis thaliana using differential mass tagging. Proteomic 6 (14), 4155-4165.

Kalmar, B., Greensmith, L., 2009. Induction of heat shock proteins for protection against oxidative stress. Adv. Drug Deliv. Rev. 61 (4), 310-318.

Kanematsu, S., Asada, K., 1990. Characteristic amino acid sequences of chloroplast and cytosol isozymes of CuZn-superoxide dismutase in spinach, rice and horsetail. Plant Cell Physiol. 31 (1), 99-112.

Kawahara, Y., de la Bastide, M., Hamilton, J.P., Kanamori, H., McCombie, W.R., Ouyang, S., Schwartz, D.C., Tanaka, T., Wu, J., Zhou, S., 2013. Improvement of the Oryza 
sativa Nipponbare reference genome using next generation sequence and optical map data. Rice 6 (1), 4.

Keith, B., Lehnhoff, E., Burns, E., Menalled, F., Dyer, W., 2015. Characterisation of Avena fatua populations with resistance to multiple herbicides. Weed Res. 55 (6), 621-630.

Keith, B.K., Burns, E.E, Bothner, B., Carey, C.C., Mazurie, A.J., Hilmer, J.K., Biyiklioglu, Budak, S., Dyer, H., WE, 2017. 2017. Intensive herbicide use has selected for constitutively elevated levels of stress-responsive mRNAs and proteins in multiple herbicide-resistant Avena fatua L. Pest Manag. Sci.

Kissen, R., Øverby, A., Winge, P., Bones, A.M., 2016. Allyl-isothiocyanate treatment induces a complex transcriptional reprogramming including heat stress, oxidative stress and plant defence responses in Arabidopsis thaliana. BMC Genomics 17 (1), 740.

Kosová, K., Vítámvás, P., Prášil, I.T., Renaut, J., 2011. Plant proteome changes under abiotic stress-contribution of proteomics studies to understanding plant stress response. J. Proteomics 74 (8), 1301-1322.

Kovacic, P., Somanathan, R., 2014. New developments in the mechanism of drug action and toxicity of conjugated imines and iminiums, including related alkaloids. Open J. Prevent. Med. 2014.

Kurepa, J., Walker, J.M., Smalle, J., Gosink, M.M., Davis, S.J., Durham, T.L., Sung, D.-Y., Vierstra, R.D., 2003. The small ubiquitin-like modifier (SUMO) protein modification system in Arabidopsis accumulation of sumol and-2 conjugates is increased by stress. J. Biol. Chem. 278 (9), 6862-6872.

Lehnhoff, E.A., Keith, B.K., Dyer, W.E., Peterson, R.K., Menalled, F., 2013. Multiple herbicide resistance in wild oat and impacts on physiology, germinability, and seed production. Agron. J. 105 (3), 854-862.

Leichert, L. I., Gehrke, F., Gudiseva, H.V., Blackwell, T., Ilbert, M., Walker, A.K., Strahler, J.R., Andrews, P.C., Jakob, U., 2008. Quantifying changes in the thiol redox proteome upon oxidative stress in vivo. Proc. Nat. Acad. Sci. USA 105 (24), 8197-8202.

Li, P., Li, Y.j., Zhang, F.j., Zhang, G.z., Jiang, X.Y., Yu, H.m., Hou, B.k., 2017. The Arabidopsis UDP-glycosyltransferases UGT79B2 and 79B3, contribute to cold, salt and drought stress tolerance via modulating anthocyanin accumulation. Plant J. 89.1, 85-103.

Liu, Y., Wu, H., Chen, H., Liu, Y., He, J., Kang, H., Sun, Z., Pan, G., Wang, Q., Hu, J., 2015. A gene cluster encoding lectin receptor kinases confers broad-spectrum and durable insect resistance in rice. Nat. Biotechnol. 33 (3), 301-305.

Losos, J.B., 2011. Convergence, adaptation, and constraint. Evolution 65 (7), 1827-1840.

Luo, X.-Y., Sunohara, Y., Matsumoto, H., 2004. Fluazifop-butyl causes membrane peroxidation in the herbicide-susceptible broad leaf weed bristly starbur (Acanthospermum hispidum). Pestic. Biochem. Physiol. 78 (2), 93-102.

Maaty, W.S., Selvig, K., Ryder, S., Tarlykov, P., Hilmer, J.K., Heinemann, J., Steffens, J., Snyder, J.C., Ortmann, A.C., Movahed, N., 2012. Proteomic analysis of Sulfolobus solfataricus during Sulfolobus turreted icosahedral virus infection. J. Proteome Res. 11 (2), 1420-1432

Macho, A.P., Zipfel, C., 2014. Plant PRRs and the activation of innate immune signaling. Mol. Cell 54 (2), 263-272.

Marone, D., Russo, M.A., Laidò, G., De Leonardis, A.M., Mastrangelo, A.M., 2013. Plant nucleotide binding site-leucine-rich repeat (NBS-LRR) genes: active guardians in host defense responses. Int. J. Mol. Sci. 14 (4), 7302-7326.

Martínez-Atienza, J., Jiang, X., Garciadeblas, B., Mendoza, I., Zhu, J.-K., Pardo, J.M., Quintero, F.J., 2007. Conservation of the salt overly sensitive pathway in rice. Plant Physiol. 143 (2), 1001-1012.

Martinez, J., Perez-Serrano, J., Bernadina, W., Rodriguez-Caabeiro, F., 2002. Expression of Hsp90, Hsp70 and Hsp60 in Trichinella species exposed to oxidative shock. J. Helminthol. 76 (03), 217-223.

Mason, K.E., Hilmer, J.K., Maaty, W.S., Reeves, B.D., Grieco, P.A., Bothner, B., Fischer, A.M., 2016. Proteomic comparison of near-isogenic barley (Hordeum vulgare L.) germplasm differing in the allelic state of a major senescence QTL identifies numerous proteins involved in plant pathogen defense. Plant Physiol. Biochem. 109, 114-127.

Minic, Z, 2008. Physiological roles of plant glycoside hydrolases. Planta 227 (4), 723.

Mittler, R., Blumwald, E., 2015. The roles of ROS and ABA in systemic acquired acclimation. Plant Cell 27 (1), 64-70.

Molina, A., Volrath, S., Guyer, D., Maleck, K., Ryals, J., Ward, E., 1999. Inhibition of protoporphyrinogen oxidase expression in Arabidopsis causes a lesion-mimic phenotype that induces systemic acquired resistance. Plant J. 17 (6), 667-678.

Morimoto, R.I., 2002. Dynamic remodeling of transcription complexes by molecular chaperones. Cell 110 (3), 281-284.

Muller, P., Ruckova, E., Halada, P., Coates, P., Hrstka, R., Lane, D., Vojtesek, B., 2013. Cterminal phosphorylation of $\mathrm{Hsp} 70$ and $\mathrm{Hsp} 90$ regulates alternate binding to cochaperones CHIP and HOP to determine cellular protein folding/degradation balances. Oncogene 32 (25), 3101-3110.

Muthuramalingam, M., Seidel, T., Laxa, M., De Miranda, S.M.N., Gärtner, F., Ströher, E., Kandlbinder, A., Dietz, K.-J., 2009. Multiple redox and non-redox interactions define 2-Cys peroxiredoxin as a regulatory hub in the chloroplast. Mol. Plant 2 (6), 1273-1288.

Naylor, J., Jana, S., 1976. Genetic adaptation for seed dormancy in Avena fatua. Can. J. Botany 54 (3-4), 306-312.

Neve, P., Powles, S., 2005. Recurrent selection with reduced herbicide rates results in the rapid evolution of herbicide resistance in Lolium rigidum. Theor. Appl. Genet. 110 (6), 1154-1166.

Nishiguchi, M., Yoshida, K., Sumizono, T., Tazaki, K., 2002. A receptor-like protein kinase with a lectin-like domain from lombardy poplar: gene expression in response to wounding and characterization of phosphorylation activity. Mol. Genet. Genomics 267 (4), 506-514.

Novatchkova, M., Budhiraja, R., Coupland, G., Eisenhaber, F., Bachmair, A., 2004. SUMO conjugation in plants. Planta 220 (1), 1-8.
Pandit, A., Rai, V., Sharma, T.R., Sharma, P.C., Singh, N.K., 2011. Differentially expressed genes in sensitive and tolerant rice varieties in response to salt-stress. J. Plant Biochem. Biotechnol. 20 (2), 149-154.

Pastor, V., Luna, E., Mauch-Mani, B., Ton, J., Flors, V., 2013. Primed plants do not forget. Environ. Exp. Bot. 94, 46-56.

Pastor, V., Balmer, A., Gamir, J., Flors, V., Mauch-Mani, B., 2014. Preparing to fight back: generation and storage of priming compounds. Front. Plant Sci. 5, 295.

Peng, Y., Abercrombie, L.L., Yuan, J.S., Riggins, C.W., Sammons, R.D., Tranel, P.J., Stewart, C.N., 2010. Characterization of the horseweed (Conyza canadensis) transcriptome using GS-FLX 454 pyrosequencing and its application for expression analysis of candidate non-target herbicide resistance genes. Pest Manag. Sci. 66 (10), 1053-1062.

Perez, I.B., Brown, P.J., 2014. The role of ROS signaling in cross-tolerance: from model to crop. Front. Plant Sci. 5, 754.

Rejeb, I.B., Pastor, V., Mauch-Mani, B., 2014. Plant responses to simultaneous biotic and abiotic stress: molecular mechanisms. Plants 3 (4), 458-475.

Savvides, A., Ali, S., Tester, M., Fotopoulos, V., 2016. Chemical priming of plants against multiple abiotic stresses: mission possible? Trends Plant Sci. 21 (4), 329-340.

Schneider, C.A., Rasband, W.S., Eliceiri, K.W., 2012. NIH image to imageJ: 25 years of image analysis. Nat. Methods 9 (7), 671-675.

Sewelam, N., Kazan, K., Schenk, P.M., 2016. Global plant stress signaling: reactive oxygen species at the cross-road. Front. Plant Sci. 7.

Shaner, D.L., 2014. Lessons learned from the history of herbicide resistance. Weed Sci. 62 (2), 427-431.

Shevchenko, A., Tomas, H., Havli, J., Olsen, J.V., Mann, M., 2006. In-gel digestion for mass spectrometric characterization of proteins and proteomes. Nat. Protoc. 1 (6), 2856-2860.

Singh, P., Zimmerli, L.Z, 2013. Lectin receptor kinases in plant innate immunity. Front. Plant Sci. 4, 124.

Smirnoff, N., 2000. Ascorbic acid: metabolism and functions of a multi-facetted molecule. Curr. Opin. Plant Biol. 3 (3), 229-235.

Suzuki, N., Miller, G., Salazar, C., Mondal, H.A., Shulaev, E., Cortes, D.F., Shuman, J.L., Luo, X., Shah, J., Schlauch, K., 2013. Temporal-spatial interaction between reactive oxygen species and abscisic acid regulates rapid systemic acclimation in plants. Plant Cell 25 (9), 3553-3569.

Suzuki, N., Rivero, R.M., Shulaev, V., Blumwald, E., Mittler, R., 2014. Abiotic and biotic stress combinations. New Phytol. 203 (1), 32-43.

Titiz, O., Tambasco-Studart, M., Warzych, E., Apel, K., Amrhein, N., Laloi, C., Fitzpatrick, T.B., 2006. PDX1 is essential for vitamin B6 biosynthesis, development and stress tolerance in Arabidopsis. Plant J. 48 (6), 933-946.

Unver, T., Bakar, M., Shearman, R.C., Budak, H., 2010. Genome-wide profiling and analysis of Festuca arundinacea miRNAs and transcriptomes in response to foliar glyphosate application. Mol. Genet. Genomics 283 (4), 397-413.

Vaid, N., Pandey, P.K., Tuteja, N., 2016. Lectin receptor-like kinases and their emerging role in abiotic stress tolerance. Abiotic Stress Response in Plants.

Vaudel, M., Barsnes, H., Berven, F.S., Sickmann, A., Martens, L., 2011. SearchGUI: An open-source graphical user interface for simultaneous OMSSA and X! Tandem searches. Proteomics 11 (5), 996-999.

Vaudel, M., Burkhart, J.M., Zahedi, R.P., Oveland, E., Berven, F.S., Sickmann, A., Martens, L., Barsnes, H., 2015. PeptideShaker enables reanalysis of MS-derived proteomics data sets. Nat. Biotechnol. 33 (1), 22-24.

Vila-Aiub, M.M., Ghersa, C.M., 2005. Building up resistance by recurrently exposing target plants to sublethal doses of herbicide. Eur. J. Agron. 22 (2), 195-207.

Vila-Aiub, M.M., Neve, P., Powles, S.B., 2009. Fitness costs associated with evolved herbicide resistance alleles in plants. New Phytol. 184 (4), 751-767.

Wagner, D., Przybyla, D., op den Camp, R., Kim, C., Landgraf, F., Lee, K.P., Würsch, M., Laloi, C., Nater, M., Hideg, E, 2004. The genetic basis of singlet oxygen-induced stress responses of Arabidopsis thaliana. Science 306 (5699), 1183-1185.

Wakeel, A., Asif, A.R., Pitann, B., Schubert, S., 2011. Proteome analysis of sugar beet (Beta vulgaris L.) elucidates constitutive adaptation during the first phase of salt stress. J. Plant Physiol. 168 (6), 519-526.

Wang, H., Wang, S., Lu, Y., Alvarez, S., Hicks, L.M., Ge, X., Xia, Y., 2011. Proteomic analysis of early-responsive redox-sensitive proteins in Arabidopsis. J. Proteome Res. 11 (1), 412-424.

Wang, M., Weiss, M., Simonovic, M., Haertinger, G., Schrimpf, S.P., Hengartner, M.O., von Mering, C., 2012. PaxDb, a database of protein abundance averages across all three domains of life. Mol. Cell. Proteomics 11 (8), 492-500.

Waszczak, C., Akter, S., Jacques, S., Huang, J., Messens, J., Van Breusegem, F., 2015. Oxidative post-translational modifications of cysteine residues in plant signal transduction. J. Exp. Bot. 66 (10), 2923-2934.

Xinlong, L., Zhang, Y., Yin, L., Lu, J., 2016. Overexpression of pathogen-induced grapevine TIR-NB-LRR gene VaRGA1 enhances disease resistance and drought and salt tolerance in Nicotiana benthamiana. Protoplasma 1-13.

Yuan, J.S., Tranel, P.J., Stewart Jr, C.N., 2007. Non-target-site herbicide resistance: a family business. Trends Plant Sci. 12 (1), 6-13.

Zhen, Y., Dhakal, P., Ungerer, M.C., 2011. Fitness benefits and costs of cold acclimation in Arabidopsis thaliana. Am. Nat. 178 (1), 44-52.

Zhou, A., Li, J., 2005. Arabidopsis BRS1 is a secreted and active serine carboxypeptidase. J. Biol. Chem. 280 (42), 35554-35561.

Zimmerli, L., Hou, B.H., Tsai, C.H., Jakab, G., Mauch-Mani, B., Somerville, S., 2008. The xenobiotic $\beta$-aminobutyric acid enhances Arabidopsis thermotolerance. Plant J. 53 (1), 144-156.

Zulet, A., Gil-Monreal, M., Zabalza, A., van Dongen, J.T., Royuela, M., 2015. Fermentation and alternative oxidase contribute to the action of amino acid biosynthesis-inhibiting herbicides. J. Plant Physiol. 175, 102-112. 